\title{
The beta Lindley distribution
}

\author{
S.M.T.K. MirMostafaee ${ }^{1}$, M. Mahdizadeh ${ }^{2}$ and Saralees Nadarajah ${ }^{3 *}$ \\ ${ }^{1}$ Department of Statistics, Faculty of Mathematical Sciences, University of Mazandaran, P.O. Box \\ 47416-1467, Babolsar, Iran \\ ${ }^{2}$ Department of Statistics, Hakim Sabzevari University, P.O. Box 397, Sabzevar, Iran \\ ${ }^{3}$ School of Mathematics, University of Manchester, Manchester M13 9PL, UK
}

\begin{abstract}
The Lindley distribution has been generalized by many authors in recent years. However, all of the known generalizations so far have restricted tail behaviors. Here, we introduce the most flexible generalization of the Lindley distribution with its tails controlled by two independent parameters. Various mathematical properties of the generalization are derived. Maximum likelihood estimators of its parameters are derived. Fisher's information matrix and asymptotic confidence intervals for the parameters are given. Finally, a real data application shows that the proposed generalization performs better than all known ones.
\end{abstract}

Keywords: Estimation, Lindley distribution, Tails.

\section{Introduction}

The Lindley distribution was first introduced by Lindley [14] whose probability density function (pdf) is

$$
f_{L}(x)=\frac{\theta^{2}}{\theta+1}(1+x) e^{-\theta x}
$$

for $x>0$ and $\theta>0$. The corresponding cumulative distribution function (cdf) is

$$
F_{L}(x)=1-\frac{\theta+1+\theta x}{\theta+1} e^{-\theta x}
$$

for $x>0$ and $\theta>0$. In recent years, this distribution has been generalized by many authors: a generalized Lindley (GL1) distribution due to Zakerzadeh and Dolati [23] with the pdf

$$
f_{G L 1}(x)=\frac{\theta^{2}(\theta x)^{\alpha-1}(\alpha+\gamma x) e^{-\theta x}}{(\gamma+\theta) \Gamma(\alpha+1)}
$$

for $x>0, \alpha>0, \theta>0$ and $\gamma>0$; a weighted Lindley (WEL) distribution due to Ghitany et al. [8] with the pdf

*Corresponding author.

E-mail addresses: m.mirmostafaee@umz.ac.ir (S.M.T.K. MirMostafaee), mahdizadeh.m@live.com ( M. Mahdizadeh), mbbsssn2@manchester.ac.uk (S. Nadarajah). 


$$
f_{W E L}(x)=\frac{\theta^{c+1}}{(\theta+c) \Gamma(c)} x^{c-1}(1+x) e^{-\theta x}
$$

for $x>0, c>0$ and $\theta>0$; a generalized Lindley (GL2) distribution due to Nadarajah et al. [16] with the pdf

$$
f_{G L 2}(x ; \alpha, \lambda)=\frac{\alpha \lambda^{2}}{1+\lambda}(1+x)\left[1-\frac{1+\lambda+\lambda x}{1+\lambda} \exp (-\lambda x)\right]^{\alpha-1} \exp (-\lambda x)
$$

for $x>0, \alpha>0$ and $\lambda>0$; an extended Lindley (EL) distribution due to Bakouch et al. [2] with the pdf

$$
f_{E L}(x)=\frac{\lambda(1+\lambda+\lambda x)^{\alpha-1}}{(1+\lambda)^{\alpha}}\left[\beta(1+\lambda+\lambda x)(\lambda x)^{\beta-1}-\alpha\right] e^{-(\lambda x)^{\beta}}
$$

for $x>0, \alpha \in(-\infty, 0) \cup\{0,1\}, \beta>0$ and $\lambda>0$; the exponential Poisson Lindley ( EPL ) distribution due to Barreto-Souza and Bakouch [3] with the pdf

$$
f_{E P L}(x)=\frac{\left.\beta \theta^{2}(1+\theta)^{2} e^{-\beta x} 3+\theta-e^{-\beta x}\right)}{\left(1+3 \theta+\theta^{2}\right)\left(1+\theta-e^{-\beta x}\right)^{3}}
$$

for $x>0, \theta>0$ and $\beta>0$; the power Lindley (PL) distribution due to Ghitany et al. [7] with the pdf

$$
f_{P L}(x)=\frac{\alpha \beta^{2}}{\beta+1}\left(1+x^{\alpha}\right) x^{\alpha-1} e^{-\beta x^{\alpha}}
$$

for $x>0, \alpha>0$ and $\beta>0$; the quasi Lindley (QL) distribution due to Shanker and Mishra [21] with the pdf

$$
f_{Q L}(x)=\frac{\theta(\alpha+x \theta)}{\alpha+1} e^{-\theta x}
$$

for $x>0, \alpha>-1$ and $\theta>0$; and the log Lindley (LL) distribution due to Gomez-Deniz et al. [9] with the pdf

$$
f_{L L}(x)=\frac{\sigma^{2}}{1+\lambda \sigma}(\lambda-\log x) x^{\sigma-1}
$$

for $0<x<1, \sigma>0$ and $\lambda>0$. We are aware of no other generalizations of the Lindley distribution.

But the Lindley distribution and its generalizations proposed so far have limited tail behavior: the Lindley distribution is restricted to have $\mathrm{fL}(0)$ fixed at a finite value and $\mathrm{fL}(\mathrm{x}) \sim \mathrm{Kxe}-\theta \mathrm{x}$ as $\mathrm{x}$ $\rightarrow \infty$, where the polynomial power of the upper tail is 1 ; the-GL1 distribution due to Zalerzadehand Dolati [23] is restricted to have fGL1(x) $\sim \mathrm{K} 1 \mathrm{x} \alpha 1$ as $\mathrm{x} \rightarrow 0$ and fGL1(x) $\sim \mathrm{K} 2 \mathrm{x} \alpha \mathrm{e} \theta \mathrm{x}$ as $\mathrm{x} \rightarrow$ $\infty$, where both polynomials are controlled by $-\alpha$; the WEL distribution due to Ghitany- et al. [8] is restricted to have fWEL(x) $\sim \mathrm{K} 1 \mathrm{xc} 1$ as $\mathrm{x} \rightarrow 0$ and fWEL(x) $\sim \mathrm{K} 2 \mathrm{xce} \theta \mathrm{x}$ as $\mathrm{x} \rightarrow \infty$, where both polynomials are controlled -by c; the GL2 distribution due to Nadarajah et al. [16] is restricted to have fGL2( $\mathrm{x}) \sim \mathrm{K} 1 \mathrm{x} \alpha 1$ as $\mathrm{x} \rightarrow 0$ and fGL2( $\mathrm{x}) \sim \mathrm{K} 2 \mathrm{xe}-\lambda \mathrm{x}$ as $\mathrm{x} \rightarrow \infty$, where the polynomial power of the upper tail- is 1; the EL distribution due -to Bakouch- $\beta$ et al. [2] is restricted to have $\mathrm{fEL}(\mathrm{x})^{-} \sim \mathrm{K} 1 \mathrm{x} \alpha+\beta 2$ as $\mathrm{x} \rightarrow 0$ and $\mathrm{fEL}(\mathrm{x})^{-\sim} \sim \mathrm{K}-2 \mathrm{x} \alpha \beta 1 \mathrm{e}(\lambda \mathrm{x})$ as $\mathrm{x} \rightarrow \infty$ if $\beta \leq 1$ and $\mathrm{fEL}(\mathrm{x}) \sim \mathrm{K} 1 \mathrm{x} \alpha$ 1 as $\mathrm{x} \rightarrow 0$ and $\mathrm{fEL}(\mathrm{x}) \sim \mathrm{K} 2 \mathrm{x} \alpha+\beta 2 \mathrm{e}(\lambda \mathrm{x})$ as $\mathrm{x} \rightarrow \infty$ if $\beta>1$, where both polynomials are controlled by $\alpha$; the EPL distribution due to Barreto-Souza and Bakouch [3] is restricted to have fEPL(0) fixed at a finite value and $\mathrm{fEPL}(\mathrm{x}) \sim \mathrm{Ke}-\beta \mathrm{x}$ as $-\mathrm{x} \rightarrow \infty$; the PL distribution due -to Ghitany $-\alpha$ et al. [7] is restricted to have $\mathrm{fPL}(\mathrm{x}) \sim \mathrm{K} 1 \mathrm{x} \alpha 1$ as $\mathrm{x} \rightarrow 0$ and $\mathrm{fPL}(\mathrm{x}) \sim \mathrm{K} 2 \mathrm{x} 2 \alpha 1 \mathrm{e} \beta \mathrm{x}$ as $\mathrm{x} \rightarrow \infty$, where both 
polynomials are controlled by $\alpha$; the QL distribution due to Shanker- and Mishra [21] is restricted to have $\mathrm{fQL}(0)$ fixed at a finite value and $\mathrm{fQL}(\mathrm{x}) \sim \mathrm{Kxe} \theta \mathrm{x}$ as $\mathrm{x} \rightarrow \infty$, where the polynomial power of the upper tail is 1; the LL distribution- due to Gomez-Deniz et al. [9] is restricted to the unit interval with $\mathrm{fLL}(\mathrm{x}) \sim-\mathrm{Kx} \sigma 1 \operatorname{logx}$ as $\mathrm{x} \rightarrow 0$ and $\mathrm{fLL}(1)$ fixed at a finite value.

We see that the Lindley distribution and all of its known generalizations have restricted tail behavior. None of them allow for the tails to behave freely. Here, we introduce the first generalization of the Lindley distribution allowing for the most flexible tails.

Let $\mathrm{G}(\mathrm{x})$ be a cdf of a continuous random variable X. Eugene et al. [5] introduced the family of beta-generated distributions defined by

$$
F(x)=\frac{1}{B(\alpha, \beta)} \int_{0}^{G(x)} t^{\alpha-1}(1-t)^{\beta-1} \mathrm{~d} t=I_{G(x)}(\alpha, \beta)
$$

for $\alpha>0$ and $\beta>0$, where

$$
B(a, b)=\int_{0}^{1} t a-1(1-t) b-1 \mathrm{~d} t
$$

denotes the beta function and

$$
I_{x}(a, b)=\frac{1}{B(a, b)} \int_{0}^{x} t^{a-1}(1-t)^{b-1} \mathrm{~d} t
$$

denotes the incomplete beta function ratio. The pdf corresponding to (11) is

$$
f(x)=\frac{1}{B(\alpha, \beta)}[G(x)]^{\alpha-1}[1-G(x)]^{\beta-1} g(x)
$$

Eugene et al. [5] studied a special case of (11) when $F(x)$ is the cdf of the normal distribution, resulting in the beta normal distribution.

The generalization of the Lindley distribution that we introduce is based on (11). Taking $G(x)$ and $g(x)$ in (11) and (12) to be the cdf and the pdf of the Lindley distribution, we obtain

$$
F(x)=I_{F L(x)}(\alpha, \beta)
$$

and

$$
f(x)=\frac{1}{B(\alpha, \beta)} F_{L}^{\alpha-1}(x)\left[1-F_{L}(x)\right]^{\beta-1} f_{L}(x)
$$

for $x>0, \alpha>0, \beta>0$ and $\theta>0$. We shall refer to the distribution given by (13) and (14) as the beta Lindley (BL) distribution. It is easy to note that $f(x) \sim K_{1} x^{\alpha-1}$ as $x \rightarrow 0$ and $f(x) \sim K_{2} x^{\beta} e^{-\beta \theta x}$ as $x \rightarrow \infty$, so the left tail is a polynomial controlled by $\alpha$, the right tail is a polynomial controlled by $\beta$ and both tails behave independently. This has not been the case with the Lindley distribution or any of its generalizations. The parameters $\alpha$ and $\beta$ in addition control the skewness and kurtosis of the BL distribution, allowing for much flexibility. Examples of other beta generalized distributions include the beta Weibull distribution due to Famoye et al. [6], the beta exponential distribution due to Nadarajah and Kotz [17], the beta gamma distribution due to Kong et al. [12], the beta Pareto distribution due to Akinsete et al. [1], the beta generalized Pareto distribution due to Mahmoudi [15] and the beta generalized half-normal geometric distribution due to Ramires et al. [20]. 
If $\mathrm{X}$ is a random variable having the $\mathrm{BL}$ distribution we shall write $\mathrm{X} \sim \operatorname{BL}(\alpha, \beta, \theta)$. The special case of the BL distribution for $\alpha=\beta=1$ is the Lindley distribution. The special case for $\beta=1$ is the GL2 distribution introduced by Nadarajah et al. [16].

Another motivation for the $\mathrm{BL}$ distribution is as follows: suppose $\mathrm{X} 1, \ldots, \mathrm{X} \beta+\alpha-1$ are independent Lindley random variables representing the failure times of the components of a $\alpha$-out-of- $\beta+\alpha-1$ system. The system will fail as soon as $\alpha$ components have failed.

Therefore, the probability that the failure time of the system say $Y$ is less than $x$ is

$$
\begin{aligned}
\operatorname{Pr}(Y \leq x) & =\operatorname{Pr}(\text { At least } \alpha \text { components out of } \beta+\alpha-1 \text { fail before time } x) \\
& =\sum_{i=\alpha}^{\alpha+\beta-1} F_{L}^{i}(x)\left[1-F_{L}(x)\right]^{\alpha+\beta-1-i} \\
& =\frac{1}{B(\alpha, \beta)} \int_{0}^{F_{L}(x)} t^{\alpha-1}(1-t)^{\beta-1} \mathrm{~d} t \\
& =I_{F_{L}(x)}(\alpha, \beta),
\end{aligned}
$$

the cdf of the BL distribution.

The rest of the paper is organized as follows. Various mathematical properties of the BL distribution are derived in Section 2. The properties discussed include shape of the pdf, expansions for the pdf and the cdf, the hazard rate function and its shape, the quantile function, moments and related measures, mean residual lifetimes, mean deviations about the mean and median, Bonferroni and Lorenz curves, and order statistics properties. In Section 3, we investigate maximum likelihood estimators (MLEs) as well as asymptotic confidence intervals for the unknown parameters. An application of the BL distribution is discussed in Section 4.

Some of the mathematical properties in Section 2 involve single infinite sums, see Sections 2.2, 2.5, 2.6, 2.7, 2.8 and 2.9. Each of these infinite sums is convergent for all parameter values. Numerical computations not reported here showed that each of these infinite sums can be truncated at 20 to yield a relative error less than 10-25 for a wide range of parameter values. This shows that the mathematical properties can be computed for most practical uses with their infinite sums truncated at twenty. The computations were performed using Maple 2015. Maple took only a fraction of a second to compute the truncated versions. The computational times for the truncated versions were significantly smaller than those for the untruncated versions.

A referee has pointed out to us that BL distribution is a particular case of the beta generalized Lindley (BGL) distribution proposed by Oluyede and Yang [18]. The BGL distribution is specified by the pdf

$$
\begin{aligned}
f_{B G L}(x)= & \frac{\alpha \theta^{2}}{B(a, b)(\theta+1)}(1+x) e^{-\theta x}\left[1-\frac{\theta+1+\theta x}{\theta+1} e^{-\theta x}\right]^{a \alpha-1} \\
& \cdot\left\{1-\left[1-\frac{\theta+1+\theta x}{\theta+1} e^{-\theta x}\right]^{\alpha}\right\}^{b-1}
\end{aligned}
$$

for $x>0, \theta>0, \alpha>0, a>0$ and $b>0$; so, the BL distribution is the particular case for $\alpha=1$.

But this paper is completely independent of Oluyede and Yang [18]. We were not aware of Oluyede and Yang [18] while writing this paper. Besides, most aspects reported in this paper are different and novel: motivation of the BL distribution as having the most flexible tail 
behavior, no such motivation was given in Oluyede and Yang [18]; motivation of the BL distribution as the distribution of the failure time of a system, no such motivation was given in Oluyede and Yang [18]; explicit expressions for quantile function, no such explicit expressions were given in Oluyede and Yang [18]; expansions for the pdf as single infinite sums, the corresponding expansions in Oluyede and Yang [18] involve double infinite sums; expansions for the cdf as single infinite sums, no such expansions were given in Oluyede and Yang [18] ; explicit expressions for moments as single infinite sums, the corresponding expressions in Oluyede and Yang [18] involve quadruple infinite sums; explicit expressions for mean deviations about the mean and median as single infinite sums, the corresponding expressions in Oluyede and Yang [18] involve double infinite sums; explicit expressions for Bonferroni and Lorenz curves as single infinite sums, the corresponding expressions in Oluyede and Yang [18] involve double infinite sums; explicit expressions for moments of order statistics, no such explicit expressions were given in Oluyede and Yang [18]; explicit expressions for moment generating function, no such explicit expressions were given in Oluyede and Yang [18]; explicit expressions for mean residual lifetime, no such explicit expressions were given in Oluyede and Yang [18]; explicit expressions for the Fisher information matrix, no such explicit expressions were given in Oluyede and Yang [18]; a more comprehensive data application with fits compared to every known generalization of the Lindley distribution; the BGL distribution does not provide a significantly better fit than the BL distribution in the data application.

\section{Mathematical properties}

Here, we derive various mathematical properties of the BL distribution: shape of the pdf (Section 2.1); expansions for the pdf and the cdf (Section 2.2); quantile function ( Section 2.3); hazard rate function and its shape (Section 2.4); moments (Section 2.5); moment generating function (Section 2.5); variance (Section 2.5); skewness (Section 2.5); kurtosis (Section 2.5); moments of the residual life (Section 2.6); mean residual life (Section 2.6) ; mean deviations about the mean and median (Section 2.7); Bonferroni curve (Section 2.8) ; Lorenz curve (Section 2.8); pdf, cdf and moments of order statistics (Section 2.9). R codes for computing some of these properties are given in the appendix.

\subsection{Shape of the pdf}

It follows from (14) that

$$
\frac{\partial}{\partial x} \log f(x)=\frac{(\alpha-1) \theta^{2}(1+x) e^{-\theta x}}{(1+\theta) F(x)}+\frac{(\beta-1) \theta}{1+\theta+\theta x}+\frac{1}{1+x}-\beta \theta,
$$

where $F(\cdot)$ is given by (13). The following results can be deduced

- If $0<\alpha<1$ or if $\alpha=1$ and $\theta \geq 1$, then the pdf is monotonically decreasing.

- If $\alpha>1$ or if $\alpha=1$ and $0<\theta<1$, then the pdf is unimodal (increasing-decreasing ) and attains its maximum at $x=x_{0}$, where

$$
\left.\frac{\partial}{\partial x} \log f(x)\right|_{x=x_{0}}=0
$$

Figure 1 plots $f(x)$ for selected parameter values. 


\subsection{Expansions for the pdf and the cdf}

Using the series expansion,

$$
(1-z)^{a-1}=\sum_{i=0}^{\infty}\left(\begin{array}{c}
a-1 \\
i
\end{array}\right)(-z)^{i}
$$

the pdf of the BL distribution can be expanded as

$$
f(x)=\frac{1}{B(\alpha, \beta)} \sum_{i=0}^{\infty}\left(\begin{array}{c}
\alpha-1 \\
i
\end{array}\right)(-1)^{i}\left[F_{L}(x)\right]^{\beta+i-1} f_{L}(x)
$$

and

$$
f(x)=\frac{1}{B(\alpha, \beta)} \sum_{i=0}^{\infty}\left(\begin{array}{c}
\beta-1 \\
i
\end{array}\right) \frac{(-1)^{i}}{\alpha+i} f_{G L 2}(x ; \alpha+i, \theta),
$$

Where $\overline{F_{L}}()=.1-F_{L}($.$) denotes the survival function. The corresponding expansions for the cdf of$ the $\mathrm{BL}$ distribution are

$$
F(x)=1-\frac{1}{B(\alpha, \beta)} \sum_{i=0}^{\infty}\left(\begin{array}{c}
\alpha-1 \\
i
\end{array}\right) \frac{(-1)^{i}}{\beta+i}\left(1+\frac{\theta x}{\theta+1}\right)^{\beta+i} e^{-(\beta+i) \theta x}
$$

and

$$
F(x)=\frac{1}{B(\alpha, \beta)} \sum_{i=0}^{\infty}\left(\begin{array}{c}
\alpha-1 \\
i
\end{array}\right) \frac{(-1)^{i}}{\alpha+i} F_{G L 2}(x ; \alpha+i, \theta)
$$

where $F_{G L}(x ; \alpha, \theta)$ denotes for the cdf corresponding to $f_{G L}(x ; \alpha, \theta)$. 

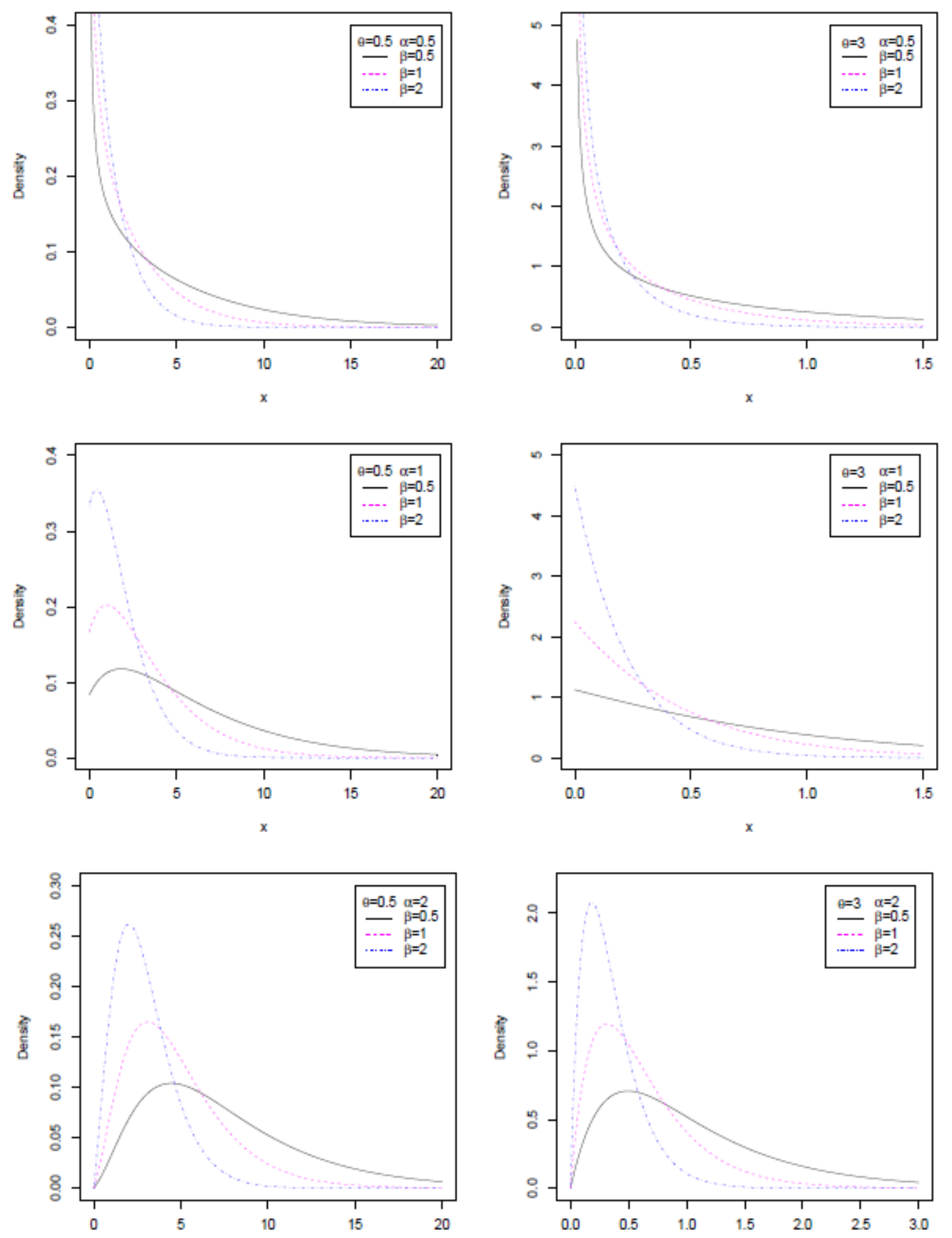

Figure $^{\mathrm{x}} 1$ : Pdfs of the BL distribution for selected $\alpha, \beta \operatorname{and}^{\mathrm{x}} \theta$. 


\subsection{Quantile function}

Jodra [11] showed that the quantile function of a Lindley random variable can be expressed as

$$
F_{L}^{-1}(y)=-1-\frac{1}{\theta}-\frac{1}{\theta} W_{-1}\left(-\frac{\theta+1}{e^{\theta+1}}(1-y)\right)
$$

where $W_{-1}(\cdot)$ is the negative branch of the Lambert $W$ function, namely the solution of $W(z) e^{W(z)}=z$. The uniqueness of the solution is guaranteed as $-\frac{1}{e}<-\frac{\theta+1}{e^{\theta+1}}(1-y)<0$, see Chapeau-Blondeau and Monir [4].

If $X \sim B L(\alpha, \beta, \theta)$, then $F_{L}(X ; \theta)$ is a beta random variable with shape parameters $\alpha$ and $\beta$. So, the quantile function of $X \sim B L(\alpha, \beta, \theta)$ is

$$
F^{-1}(p)=-1-\frac{1}{\theta}-\frac{1}{\theta} W_{-1}\left(-\frac{\theta+1}{e^{\theta+1}}\left\{1-I_{p}^{-1}(\alpha, \beta)\right\}\right)
$$

where $I_{y}{ }^{-1}(\alpha, \beta)$ denotes the inverse function of $I_{y}(\alpha, \beta)$.

\subsection{Hazard rate function}

The hazard rate function of the BL distribution is

$$
h(x)=\frac{(\theta+1)^{-1} \theta^{2}(1+x) e^{-\beta \theta x}}{B(\alpha, \beta)-I_{\left\{1-\frac{\theta+1+\theta x}{\theta+1} e^{-\theta x}\right\}}(\alpha, \beta)}\left[1-\left(1+\frac{\theta x}{\theta+1}\right) e^{-\theta x}\right]^{\alpha-1}\left(1+\frac{\theta x}{\theta+1}\right)^{\beta-1} .
$$

It follows from (16) that

$$
\frac{\partial}{\partial x} \log h(x)=\frac{\partial}{\partial x} \log f(x)+h(x)
$$

The following results can be deduced

- If $0<\alpha<1$, then the hazard rate function is decreasing-increasing (bathtub shaped) and attains its minimum at $x=x_{0}$, where

$$
\left.\frac{\partial}{\partial x} \log h(x)\right|_{x=x_{0}}=0
$$

- If $\alpha \geq 1$, then the hazard rate function is monotonically increasing.

Figure 2 plots $h(x)$ for selected parameter values.

\subsection{Moments, moment generating function, skewness and kurtosis}

Let $X \sim B L(\alpha, \beta, \theta)$. The moment generating function and the $k$ th moment of $X$ can be derived as $M_{X}(t)=\frac{1}{B(\alpha, \beta)} \sum_{i=0}^{\infty}\left(\begin{array}{c}\alpha-1 \\ i\end{array}\right) \frac{(-1)^{i}}{\beta+i-t / \theta}\left\{1-\frac{t e^{(\beta+i-t / \theta)(\theta+1)} \Gamma(\beta+i,(\theta+1)(\beta+i-t / \theta))}{\theta[(\theta+1)(\beta+i-t / \theta)]^{\beta+i}}\right\}$ 

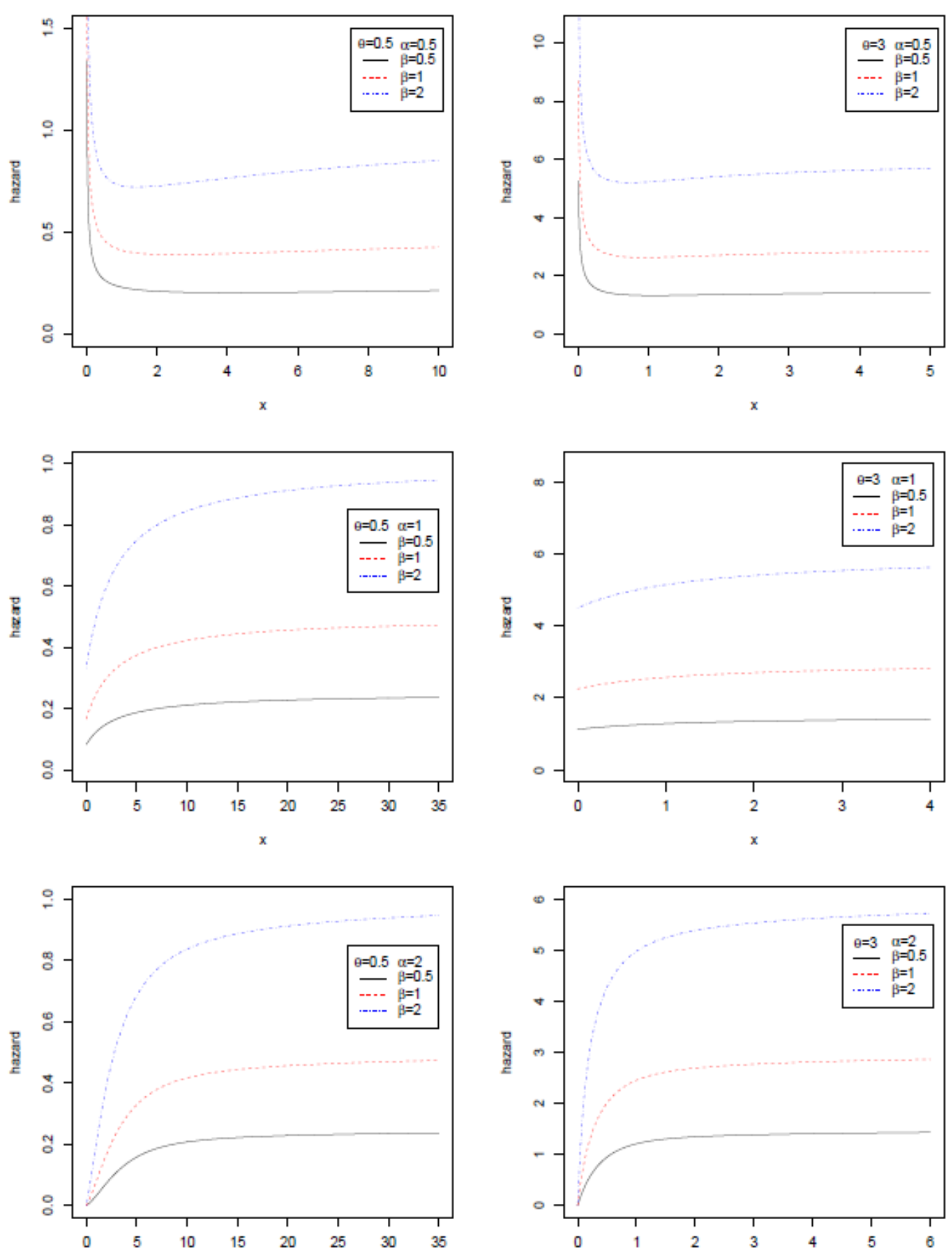

Figure 2: Hazard $^{\mathrm{x}}$ rate function of the BL distribution for selected ${ }^{\mathrm{x}} \alpha, \beta$ and $\theta$.

and

$E\left(X^{k}\right)=\frac{1}{B(\alpha, \beta)} \sum_{i=0}^{\infty} \sum_{j=0}^{k}\left(\begin{array}{c}\alpha-1 \\ i\end{array}\right)\left(\begin{array}{c}k \\ j\end{array}\right) \frac{(-1)^{i+k-j}(\theta+1)^{k}}{\theta^{k}(\beta+i)}\left\{1+\frac{j e^{(\beta+i)(\theta+1)} \Gamma(\beta+i+j,(\theta+1)(\beta+i))}{[(\beta+i)(\theta+1)]^{\beta+i+j}}\right\}$ provided that $t<\beta \theta$, where $\Gamma(a, x)=\int_{x}^{\infty} t^{a-1} e^{-t} \mathrm{~d} t$ denotes the incomplete gamma function. If $\alpha$ and $\beta$ are integers then the latter simplifies to 
$E\left(X^{k}\right)=\frac{\theta^{-k}}{B(\alpha, \beta)} \sum_{i=0}^{\alpha-1} \sum_{j=0}^{\beta+i-1}\left(\begin{array}{c}\alpha-1 \\ i\end{array}\right)\left(\begin{array}{c}\beta+i-1 \\ j\end{array}\right) \frac{(-1)^{i} \Gamma(k+j+1)}{(\theta+1)^{j+1}(\beta+i)^{k+j+1}}\left(\theta+\frac{k+j+1}{\beta+i}\right)$
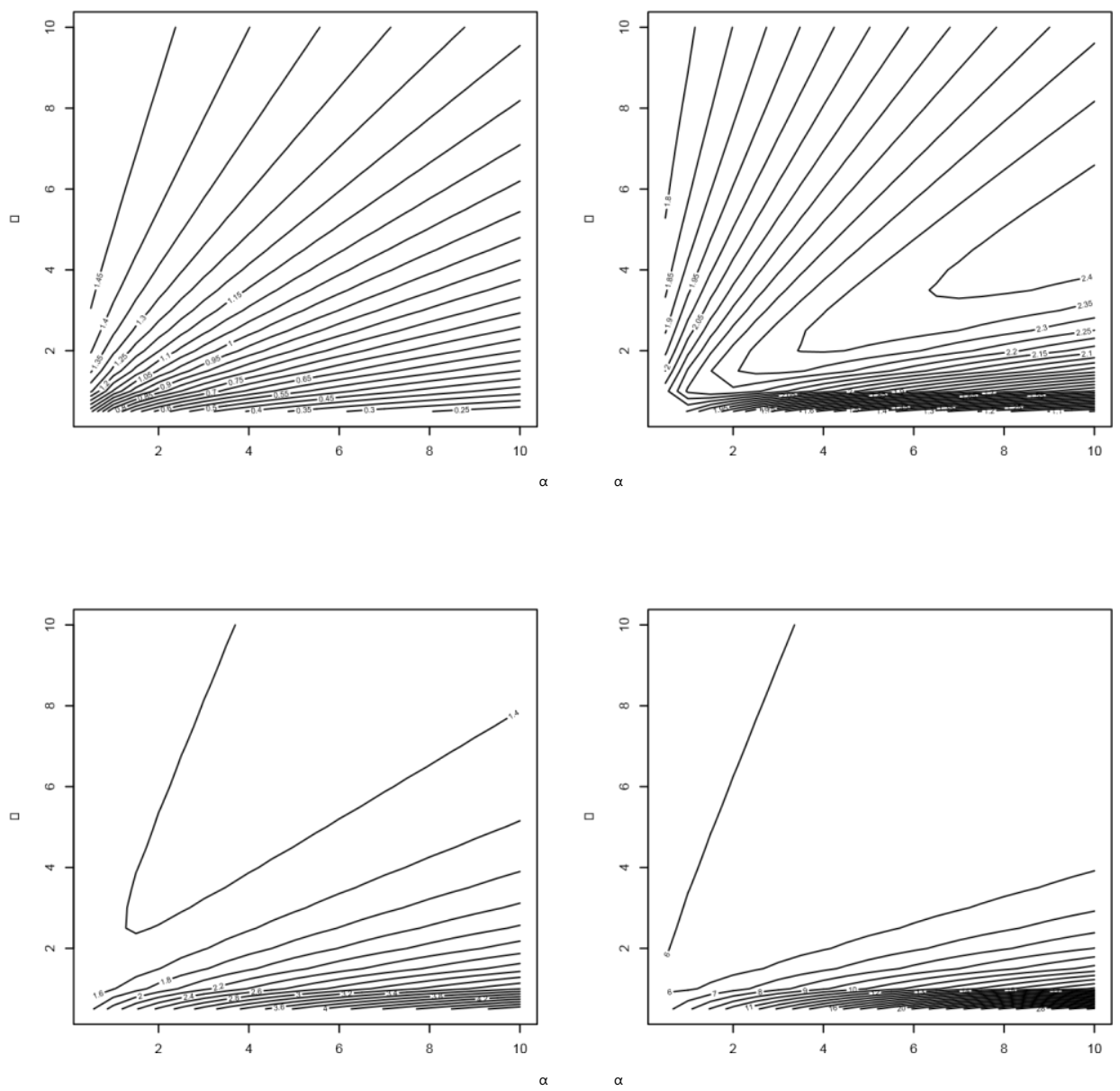

Figure 3: Mean, variance, skewness and kurtosis of the BL distribution versus $\alpha=0.5,1, \ldots, 10$ and $\beta$ $=0.5,1, \ldots, 10$ for $\theta=1$.

The variance, skewness and kurtosis of $X \sim B L(\alpha, \beta, \theta)$ can be computed using the relations:

$$
\begin{aligned}
& \left.\operatorname{Var}(X)=E \quad X^{2}\right)-[E(X)]^{2}, \\
& \operatorname{Skewness}(X)=\frac{\left.\left.E X^{3}\right)-3 E(X) E X^{2}\right)+2[E(X)]^{3}}{[\operatorname{Var}(X)]^{\frac{3}{2}}}, \\
& \operatorname{Kurtosis}(X)=\frac{\left.\left.\left.E X^{4}\right)-4 E(X) E X^{3}\right)+6 E X^{2}\right)[E(X)]^{2}-3[E(X)]^{4}}{[\operatorname{Var}(X)]^{2}} .
\end{aligned}
$$

Figure 3 shows how mean, variance, skewness and kurtosis vary with respect to $\alpha$ and $\beta$ when $\theta=$ 1. We can observe the following: mean is a decreasing function of $\alpha$ and an increasing function of $\beta$; variance is a decreasing function of $\alpha$ and a decreasing function of $\beta$; skewness is an increasing 
function of $\alpha$ and a decreasing function of $\beta$; kurtosis is an increasing function of $\alpha$ and a decreasing function of $\beta$.

\subsection{Mean residual lifetime}

Given that a component survives up to time $t>0$, the residual life is the period beyond $t$ until the failure time. It is defined by the conditional random variable $X-t \mid X>t$. It is well-known that the mean residual life function and ratio of two consecutive moments of residual life determine the distribution uniquely.

Standard calculations show that the $r$ th order moment of the residual life $\mu_{r}(t)=E\left[(X-t)^{r} \mid X\right.$ $>t$ ] for the BL distribution can be expressed as

$$
\begin{aligned}
\mu_{r}(t)= & \frac{1}{B(\alpha, \beta) \bar{F}(t)} \sum_{i=0}^{\infty} \sum_{j=0}^{r} \sum_{\ell=0}^{j}\left(\begin{array}{c}
\alpha-1 \\
i
\end{array}\right)\left(\begin{array}{l}
r \\
j
\end{array}\right)\left(\begin{array}{l}
j \\
\ell
\end{array}\right) \\
& \cdot \frac{(-1)^{i+r-\ell}}{\theta^{j}}\left(\frac{e^{\theta+1}}{\theta+1}\right)^{\beta+i} t^{r-j}(1+\theta)^{j-\ell} \xi(1+\theta(1+t), \ell+\beta+i, \beta+i),
\end{aligned}
$$

where $\xi(x, a, b)=b^{-1} h x^{a} e^{-b x}+b^{-a}(a-b) \Gamma(a, b x)$ i. The particular case $r=1$ gives the mean residual lifetime as

$$
\begin{aligned}
\mu_{1}(t)=-t+ & \frac{1}{\theta B(\alpha, \beta) \bar{F}(t)} \sum_{i=0}^{\infty}\left(\begin{array}{c}
\alpha-1 \\
i
\end{array}\right)(-1)^{i}\left(\frac{e^{\theta+1}}{\theta+1}\right)^{\beta+i} \xi(1+\theta(1+t), \beta+i+1, \beta+i) \\
& -\frac{\theta+1}{\theta B(\alpha, \beta) \bar{F}(t)} \sum_{i=0}^{\infty}\left(\begin{array}{c}
\alpha-1 \\
i
\end{array}\right)(-1)^{i}\left(\frac{e^{\theta+1}}{\theta+1}\right)^{\beta+i} \xi(1+\theta(1+t), \beta+i, \beta+i) .
\end{aligned}
$$

Figure 4 plots $\mu_{1}(t)$ for selected $\alpha$ and $\beta$.

In reliability, the reversed residual life is defined as the conditional random variable $t-X \mid X \leq t$. This random variable is the time elapsed from the failure of a component given that its life is less than or equal to $t$. Expressions for the $r$ th order moment of the reversed residual lifetime for the BL distribution can be derived similarly.

\subsection{Mean deviations}

Two important measures of spread in a population are called the mean deviation about the mean (when the distribution is symmetric) and the mean deviation about the median
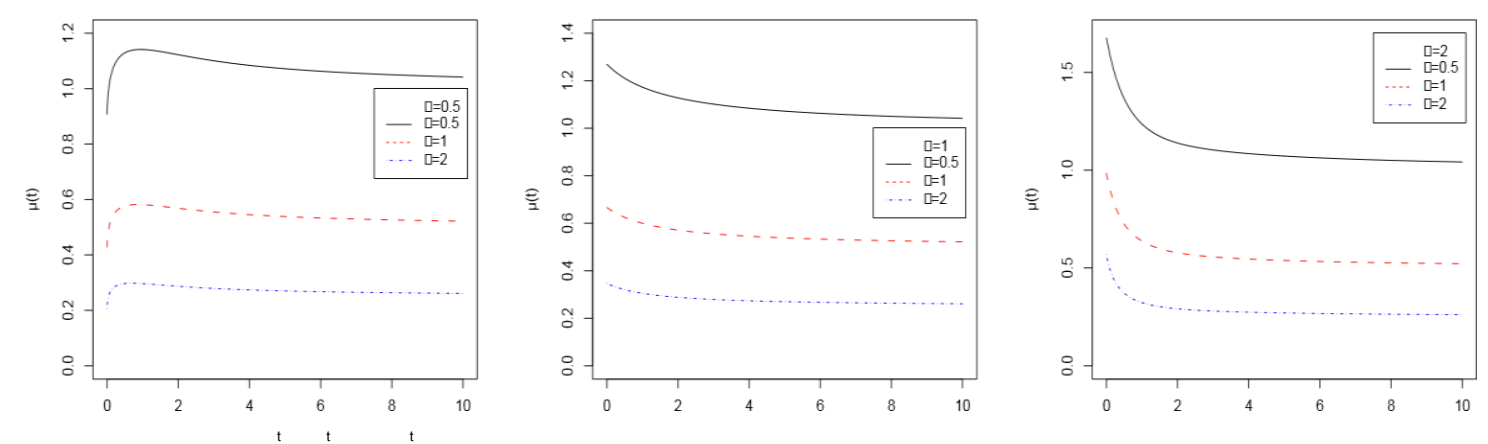

Figure 4: Mean residual life function of the $\mathrm{BL}$ distribution for some $\beta$ and $\alpha$ when $\theta=2$. 
(when the distribution is skewed). If $X \sim B L(\alpha, \beta, \theta)$, then the mean deviation about the mean and the mean deviation about the median can be given by

$$
M D(\mu)=E\{|X-\mu|\}=\int_{0}^{\infty}|X-\mu| f(x) \mathrm{d} x
$$

and

$$
M D(m)=E\{|X-m|\}=\int_{0}^{\infty}|X-m| f(x) \mathrm{d} x,
$$

respectively, where $\mu$ and $m$ denote, respectively, the mean and the median of the BL distribution. Standard calculations show that

$$
\begin{aligned}
M D(\mu)= & \frac{-2 \mu}{B(\alpha, \beta)} \sum_{i=0}^{\infty}\left(\begin{array}{c}
\alpha-1 \\
i
\end{array}\right) \frac{(-1)^{i}}{\beta+i}\left(1+\frac{\theta \mu}{\theta+1}\right)^{\beta+i} e^{-(\beta+i) \theta \mu} \\
& +\frac{2}{\theta B(\alpha, \beta)} \sum_{i=0}^{\infty}\left(\begin{array}{c}
\alpha-1 \\
i
\end{array}\right)(-1)^{i}\left(\frac{e^{\theta+1}}{\theta+1}\right)^{\beta+i} \xi(1+\theta(1+\mu), \beta+i+1, \beta+i) \\
& -\frac{2(\theta+1)}{\theta B(\alpha, \beta)} \sum_{i=0}^{\infty}\left(\begin{array}{c}
\alpha-1 \\
i
\end{array}\right)(-1)^{i}\left(\frac{e^{\theta+1}}{\theta+1}\right)^{\beta+i} \xi(1+\theta(1+\mu), \beta+i, \beta+i)
\end{aligned}
$$

and

$$
\begin{aligned}
M D(m)= & m-\mu-\frac{2 m}{B(\alpha, \beta)} \sum_{i=0}^{\infty}\left(\begin{array}{c}
\alpha-1 \\
i
\end{array}\right) \frac{(-1)^{i}}{\beta+i}\left(1+\frac{\theta m}{\theta+1}\right)^{\beta+i} e^{-(\beta+i) \theta m} \\
& +\frac{2}{\theta B(\alpha, \beta)} \sum_{i=0}^{\infty}\left(\begin{array}{c}
\alpha-1 \\
i
\end{array}\right)(-1)^{i}\left(\frac{e^{\theta+1}}{\theta+1}\right)^{\beta+i} \xi(1+\theta(1+m), \beta+i+1, \beta+i) \\
& -\frac{2(\theta+1)}{\theta B(\alpha, \beta)} \sum_{i=0}^{\infty}\left(\begin{array}{c}
\alpha-1 \\
i
\end{array}\right)(-1)^{i}\left(\frac{e^{\theta+1}}{\theta+1}\right)^{\beta+i} \xi(1+\theta(1+m), \beta+i, \beta+i) .
\end{aligned}
$$

\subsection{Bonferroni and Lorenz curves}

If $X \sim B L(\alpha, \beta, \theta)$ then Bonferroni curve is the plot of $B(F(x))$ versus $x$, where

$$
B(F(x))=\frac{1}{\mu F(x)} \int_{0}^{x} t f(t) \mathrm{d} t,
$$

where $\mu$ denotes the mean of the BL distribution. Standard calculations show that

$$
\begin{aligned}
B(F(x))= & \frac{1}{F(x)} \\
& -\frac{1}{\mu \theta B(\alpha, \beta) F(x)} \sum_{i=0}^{\infty}\left(\begin{array}{c}
\alpha-1 \\
i
\end{array}\right)(-1)^{i}\left(\frac{e^{\theta+1}}{\theta+1}\right)^{\beta+i} \xi(1+\theta(1+x), \beta+i+1, \beta+i) \\
& +\frac{\theta+1}{\mu \theta B(\alpha, \beta) F(x)} \sum_{i=0}^{\infty}\left(\begin{array}{c}
\alpha-1 \\
i
\end{array}\right)(-1)^{i}\left(\frac{e^{\theta+1}}{\theta+1}\right)^{\beta+i} \xi(1+\theta(1+x), \beta+i, \beta+i) .
\end{aligned}
$$

The Lorenz curve of $X \sim B L(\alpha, \beta, \theta)$ is the plot of $L(F(x))$ versus $x$, where $L(F(x))=F(x) B(F(x))$. 


\subsection{Order statistics}

Suppose $X_{1}, X_{2}, \ldots, X_{n}$ is a random sample of size $n$ from the BL distribution. Suppose further $X_{1: n} \leq$ $X_{2: n} \leq \cdots \leq X_{n: n}$ are the corresponding order statistics. Then, the pdf, the cdf and the survival function of $m$ th order statistic $X_{m: n}=Y_{m}$ say can be expressed as

$$
\begin{gathered}
f_{Y_{m}}(y)=\frac{f(y)}{B(m, n-m+1)} \sum_{s=0}^{m-1}\left(\begin{array}{c}
m-1 \\
s
\end{array}\right)(-1)^{s}[\bar{F}(y)]^{n-m+s}, \\
F_{Y_{m}}(y)=\sum_{\ell=m}^{n} \sum_{s=0}^{\ell}\left(\begin{array}{l}
n \\
\ell
\end{array}\right)\left(\begin{array}{l}
\ell \\
s
\end{array}\right)(-1)^{s}[\bar{F}(y)]^{n-\ell+s}
\end{gathered}
$$

and

$$
\bar{F}_{Y_{m}}(y)=\sum_{\ell=0}^{m-1} \sum_{s=0}^{\ell}\left(\begin{array}{l}
n \\
\ell
\end{array}\right)\left(\begin{array}{l}
\ell \\
s
\end{array}\right)(-1)^{s}[\bar{F}(y)]^{n-\ell+s},
$$

respectively.

These expressions can be simplified using the following from Gradshteyn and Ryzhik [10], Section 0.314: for any positive integer $j$, we have

$$
\left(\sum_{i=0}^{\infty} a_{i} x^{i}\right)^{j}=\sum_{i=0}^{\infty} c_{j, i} x^{i}
$$

where the coefficients $c_{j, i}$ for $i=1,2, \ldots$ can be obtained from the recurrence relation

$$
c_{j, i}=\left(i a_{0}\right)^{-1} \sum_{q=1}^{i}(j q-i+q) a_{q} c_{j, i-q}
$$

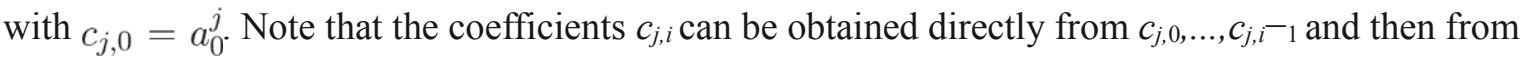
the coefficients $a_{0}, \ldots, a_{i}$ of the original power series.

Using (20) and (21), the expressions in (17), (18) and (19) can be simplified to

$$
\begin{array}{r}
f_{Y_{m}}(y)=\frac{\theta^{2}(1+y)(1-u)^{\alpha-1} u^{(n-m+1) \beta-1} e^{-\theta y}}{(\theta+1) B(m, n-m+1)} \sum_{s=0}^{m-1} \sum_{i=0}^{\infty} \frac{\left.m^{m-1}\right)(-1)^{s}}{[B(\alpha, \beta)]^{n-m+s+1}} c_{n-m+s, i} u^{\beta s+i} \\
F_{Y_{m}}(y)=\sum_{\ell=m}^{n} \sum_{s=0}^{\ell} \sum_{i=0}^{\infty}\left(\begin{array}{l}
n \\
\ell
\end{array}\right)\left(\begin{array}{l}
\ell \\
s
\end{array}\right) \frac{(-1)^{s}}{[B(\alpha, \beta)]^{n-\ell+s}} c_{n-\ell+s, i} u^{(n-\ell+s) \beta+i}
\end{array}
$$

and

$$
\bar{F}_{Y_{m}}(y)=\sum_{\ell=0}^{m-1} \sum_{s=0}^{\ell} \sum_{i=0}^{\infty}\left(\begin{array}{l}
n \\
\ell
\end{array}\right)\left(\begin{array}{l}
\ell \\
s
\end{array}\right) \frac{(-1)^{s}}{[B(\alpha, \beta)]^{n-\ell+s}} c_{n-\ell+s, i} u^{(n-\ell+s) \beta+i},
$$

respectively, where $u=1-F_{L}(y), w_{i}=\left(\begin{array}{c}\alpha-1 \\ i\end{array}\right) \frac{(-1)^{i}}{\beta+i}$ and the constants $c_{n}{ }^{-}{ }^{++s, i}$ can be calculated from (21) as 


$$
c_{n-m+s, i}=\left(i w_{0}\right)^{-1} \sum_{q=1}^{i}[(n-m+s) q-i+q] w_{q} c_{n-m+s, i-q}
$$

where $c_{n-m+s, 0}=w_{0}^{n-m+s}$. So, the coefficients $c_{n}{ }^{-{ }^{+}+s, i}$ can be obtained from $c_{n}{ }^{-{ }^{+}+s, 0}$, $\ldots, c_{n}{ }^{-}{ }^{m+s, i}{ }_{1}$ and then from $w_{0}, \ldots, w_{i}$. Finally, the $k$ th moment of $Y_{m}$ can be expressed as

$$
\begin{aligned}
E\left(Y_{m}^{k}\right)=k \sum_{s=n-m+1}^{n} \sum_{i=0}^{\infty} \sum_{j=0}^{k-1}\left(\begin{array}{c}
s-1 \\
n-m
\end{array}\right)\left(\begin{array}{c}
n \\
s
\end{array}\right)\left(\begin{array}{c}
k-1 \\
j
\end{array}\right) \\
\cdot \frac{(-1)^{s-n+m-2+k-j} e^{(s \beta+i)(1+\theta)} c_{s, i}}{[B(\alpha, \beta)]^{s}(1+\theta)^{s \beta+i+j-k} \theta^{k}(s \beta+i)^{s \beta+i+j+1}} \\
\cdot \Gamma(s \beta+i+j+1,(s \beta+i)(1+\theta)) .
\end{aligned}
$$

\section{Maximum likelihood estimation of the parameters}

In this section, we discuss maximum likelihood estimation of the parameters of the BL distribution. Suppose that $\mathrm{x} 1, \ldots, \mathrm{xn}$ is a random sample of size $\mathrm{n}$ from the $\mathrm{BL}$ distribution. The log-likelihood function is

$$
\begin{aligned}
\log L= & -n \log B(\alpha, \beta)+(\alpha-1) \sum_{i=1}^{n} \log \left[1-\left(1+\frac{\theta x_{i}}{\theta+1}\right) e^{-\theta x_{i}}\right] \\
+ & (\beta-1) \sum_{i=1}^{n} \log \left(1+\frac{\theta x_{i}}{\theta+1}\right)+n \log \left(\frac{\theta^{2}}{\theta+1}\right)+\sum_{i=1}^{n} \log \left(1+x_{i}\right) \\
& -\beta \theta \sum_{i=1}^{n} x_{i} .
\end{aligned}
$$

The MLEs of $\alpha, \beta$ and $\theta$ say $\alpha, \beta$ and $\theta$, respectively, can be obtained as the solutions of the non-linear equations $\mathrm{b} \mathrm{b} \mathrm{b}$

$$
\begin{aligned}
& \frac{\partial}{\partial \alpha} \log L=-n \Psi(\alpha)+n \Psi(\alpha+\beta)+\sum_{i=1}^{n} \log \left[1-\left(1+\frac{\theta x_{i}}{\theta+1}\right) e^{-\theta x_{i}}\right]=0 \\
& \frac{\partial}{\partial \beta} \log L=-n \Psi(\beta)+n \Psi(\alpha+\beta)+\sum_{i=1}^{n} \log \left(1+\frac{\theta x_{i}}{\theta+1}\right)-\theta \sum_{i=1}^{n} x_{i}=0 \\
& \frac{\partial}{\partial \theta} \log L=(\alpha-1) \sum_{i=1}^{n} \frac{\left[\left(1+\frac{\theta x_{i}}{\theta+1}\right)-\frac{1}{(\theta+1)^{2}}\right] x_{i} e^{-\theta x_{i}}}{1-\left(1+\frac{\theta x_{i}}{\theta+1}\right) e^{-\theta x_{i}}}+(\beta-1) \sum_{i=1}^{n} \frac{\frac{x_{i}}{(\theta+1)^{2}}}{1+\frac{\theta x_{i}}{\theta+1}} \\
&+n-\beta \sum_{i=1}^{n} x_{i}=0
\end{aligned}
$$

where $\Psi(\mathrm{t})=\frac{\mathrm{d}}{\mathrm{d} t} \log \Gamma(\mathrm{t})$ denotes the digamma function. Alternatively, the MLEs can be obtained by maximizing (22) numerically. We shall use the latter approach in Section 4. The maximization was performed by using the $\mathrm{nlm}$ function in R (R Development Core Team [19]). In Section 4 , the function $\mathrm{nIm}$ was executed with the following initial values: $\alpha=0.01,0.02, \ldots, 5$, $\beta=0.01,0.02, \ldots, 5$ and $\theta=0.01,0.02, \ldots, 5$. Each time the function converged the solutions for the MLEs were unique. The function did not converge about five percent of the time. 
Interval estimation and hypothesis testing of $(\alpha, \beta, \theta)$ requires the expected Fisher information matrix. The elements of this matrix say $\mathbf{I}=\left(I_{i j}\right), i, j=1,2,3$ about $(\alpha, \beta, \theta)$ based on a single observation can be calculated as

$$
\begin{aligned}
& I_{11}=\Psi^{\prime}(\alpha)-\Psi^{\prime}(\alpha+\beta), \\
& I_{22}=\Psi^{\prime}(\beta)-\Psi^{\prime}(\alpha+\beta), \\
& I_{12}=I_{21}=-\Psi^{\prime}(\alpha+\beta), \\
& I_{13}=I_{31}=-A_{1}, \\
& I_{23}=I_{32}=\mu-A_{2}, \\
& I_{33}=\frac{2}{\theta^{2}}-\frac{1}{(\theta+1)^{2}}-(\alpha-1) A_{3}-(\beta-1) A_{4},
\end{aligned}
$$

where $\Psi^{\prime}(t)=\frac{\mathrm{d}}{\mathrm{d} t} \Psi(t)$ denotes the trigamma function,

$$
\begin{gathered}
A_{1}=\frac{\beta}{\alpha-1} E(Y)-\frac{\alpha+\beta-1}{(\alpha-1)(\theta+1)^{2}} \varphi(\alpha-1, \beta, \theta, 1,1) \\
A_{2}=\frac{\alpha+\beta-1}{(\beta-1)(\theta+1)^{2}} \varphi(\alpha, \beta-1, \theta, 1,1) \\
\left.A_{3}=\frac{2(\alpha+\beta-1)}{(\theta+1)^{3}} \varphi(\alpha-1, \beta, \theta, 1,1)-\beta E Y^{2}\right)+\frac{2(\alpha+\beta-1)}{(\theta+1)^{2}} \varphi(\alpha-1, \beta, \theta, 2,1) \\
\left.-\beta \theta E(Y)-\frac{\beta(\beta+1)}{\alpha-2} E Z^{2}\right)-\frac{(\alpha+\beta-1)(\alpha+\beta-2)}{(\alpha-2)(\theta+1)^{4}} \varphi(\alpha-2, \beta, \theta, 2,2) \\
+\frac{2 \beta(\alpha+\beta-1)}{(\alpha-2)(\theta+1)^{2}} \varphi(\alpha-2, \beta+1, \theta, 2,1)
\end{gathered}
$$

and

$$
A_{4}=-\frac{(\alpha+\beta-1)(\alpha+\beta-2)}{\beta(\theta+1)^{3}}\left[2 \varphi(\alpha, \beta-2, \theta, 1,2)+\frac{2 \theta+1}{\theta+1} \varphi(\alpha, \beta-2, \theta, 2,2)\right],
$$

where $Y \sim B L(\alpha-1, \beta+1), Z \sim B L(\alpha-2, \beta+2)$ and

$$
\begin{aligned}
\varphi(\alpha, \beta, \theta, k, r)=\frac{1}{B(\alpha, \beta)} \sum_{i=0}^{\infty} \sum_{j=0}^{k}\left(\begin{array}{c}
\alpha-1 \\
i
\end{array}\right)\left(\begin{array}{c}
k \\
j
\end{array}\right) \frac{(-1)^{i+k-j}(\theta+1)^{k}}{\theta^{k}(\beta+i+r)} \\
\cdot\left[1+\frac{(j-r) e^{(\theta+1)(\beta+i+r)} \Gamma(\beta+i+j,(\theta+1)(\beta+i+r))}{[(\theta+1)(\beta+i+r)]^{\beta+i+j}}\right] .
\end{aligned}
$$

Note that $\varphi(\alpha, \beta, \theta, k, 0)=E\left(X^{k}\right)$ for $X \sim B L(\alpha, \beta, \theta)$.

Under certain regularity conditions (see, for example, Lehmann and Casella [13], pages 461$463)$, the asymptotic joint distribution of the $\operatorname{MLEs}(\widehat{\alpha}, \widehat{\beta}, \widehat{\theta})$ can be stated as

$$
\left.\sqrt{n}\left[\begin{array}{c}
\widehat{\alpha}-\alpha \\
\widehat{\beta}-\beta \\
\widehat{\theta}-\theta
\end{array}\right] \stackrel{D}{\longrightarrow} N_{3} \quad \mathbf{0}, \mathbf{I}^{-1}\right)^{\prime}
$$


where $\stackrel{D}{\rightarrow}$ denotes convergence in distribution and $\mathbf{I}^{-1}$ is the inverse of the Fisher information matrix I with

$$
\frac{1}{n} \mathbf{I}^{-1}=\frac{1}{n}\left[\begin{array}{ccc}
I_{11} & I_{12} & I_{13} \\
I_{12} & I_{22} & I_{23} \\
I_{13} & I_{23} & I_{33}
\end{array}\right]^{-1}=\frac{1}{n}\left[\begin{array}{ccc}
\operatorname{Var}(\widehat{\alpha}) & \operatorname{Cov}(\widehat{\alpha}, \widehat{\beta}) & \operatorname{Cov}(\widehat{\alpha}, \widehat{\theta}) \\
\operatorname{Cov}(\widehat{\alpha}, \widehat{\beta}) & \operatorname{Var}(\widehat{\beta}) & \operatorname{Cov}(\widehat{\beta}, \widehat{\theta}) \\
\operatorname{Cov}(\alpha, \widehat{\theta}) & \operatorname{Cov}(\widehat{\beta}, \widehat{\theta}) & \operatorname{Var}(\widehat{\theta})
\end{array}\right] .
$$

The unknown parameters in the elements of thebmatrix $\mathbf{I}^{-1}$ can be replaced by their corresponding MLEs. The asymptotic equal tailed 100(1-p) percent confidence intervals for the parameters $\alpha, \beta$ and $\theta$ are

$$
\widehat{\alpha} \pm z_{p / 2} \sqrt{\widehat{\operatorname{Var}(\widehat{\alpha})}}, \quad \widehat{\beta} \pm z_{p / 2} \sqrt{\widehat{\operatorname{Var}(\widehat{\beta})}}, \quad \widehat{\theta} \pm z_{p / 2} \sqrt{\widehat{\operatorname{Var}(\widehat{\theta})}}
$$

respectively, where $z_{a}$ denotes the $100 a$ percentile of the standard normal random variable.

\section{Application}

Here, we illustrate the power of the BL distribution by using a real data set taken from Ugarte et al. [22]:

130126139126124149124138138140127140124124121

125134121125126122137146127124142122126124126

121138124126137122131128122144

The data are the scores of the Stanford-Binet intelligence quotient (IQ) test for forty randomly selected, gifted and talented students. Some summary statistics of the data are: the minimum is 121 , the first quartile is 124 , the median is 126 , the mean is 129.8 , the third quartile is 137.2 , the maximum is 149 , the skewness is 0.790 , and the kurtosis is -0.729 . We shall refer to the data as IQ data.

We fitted the following eleven distributions to the IQ data: the Lindley distribution specified by the pdf (1); the GL1 distribution specified by the pdf (3); the WEL distribution specified by the pdf (4); the GL2 distribution specified by the pdf (5); the EL distribution specified by the pdf (6); the EPL distribution specified by the pdf (7); the PL distribution specified by the pdf (8); the QL distribution specified by the pdf (9); the BGL distribution specified by the pdf (15); the proposed BL distribution specified by the pdf (14); the Weibull distribution specified by the pdf

$$
f(x)=a b^{a} X^{a-1} \exp \left[-(b x)^{a}\right]
$$

for $x>0, a>0$ and $b>0$; the gamma distribution specified by the pdf

$$
f(x)=\frac{b^{a}}{\Gamma(a)} x^{a-1} \exp (-b x)
$$

for $x>0, a>0$ and $b>0$. Note that these distributions include all of the known generalizations of the Lindley distribution. The BGL distribution has four parameters. The GL1, EL and BL distributions have each three parameters. The WEL, GL2, EPL, PL, QL, Weibull and gamma distributions have each two parameters. The Lindley distribution has one parameter. The LL distribution in (10) was not fitted since it is defined on the unit interval. 
Each distribution was fitted by the method of maximum likelihood. The BL distribution was fitted by following the details in Section 3 .

Table 1 lists the parameter estimates, their standard errors (computed by inverting the expected information matrices), the negative log-likelihood values, the values of the Akaike information criterion (AIC), the values of the Bayesian information criterion (BIC), and the p-values based on the Kolmogorov-Smirnov statistic.

We see that the BGL distribution has the largest log-likelihood value, but the BL distribution has the smallest AIC value, the smallest BIC value, and the largest $p$-value in spite of the fact that the BGL distribution has one more parameter and two other fitted distributions have the same number of parameters. Use of the likelihood ratio test shows that the BGL distribution does not improve significantly on the fit of the BL distribution. The BGL distribution however has the second largest log-likelihood value, the second smallest AIC value, the fourth smallest BIC value, and the second largest p-value. The WEL and gamma distributions have the third largest log-likelihood value, the third smallest AIC value, the second smallest BIC value, and the third largest p-value. The EL distribution has the smallest log-likelihood value, the largest AIC value, the largest BIC value, and the smallest p-value. The EPL distribution has the second smallest log-likelihood value, the second largest AIC value, the second largest BIC value, and the second smallest $p$ - value.

Thus we can conclude that the BL distribution provides the best fit among the distributions considered here for the IQ data. The second best fit is by the BGL distribution. The third best fit is by the WEL and gamma distributions. The worst fit is by the EL distribution. The second worst fit is by the EPL distribution. The density and probability plots shown in Figures 5 and 6 confirm these observations. The fitted pdf of the BL distribution best captures the empirical histogram. The plotted points for the BL distribution are most closest to the diagonal line in the probability plot.

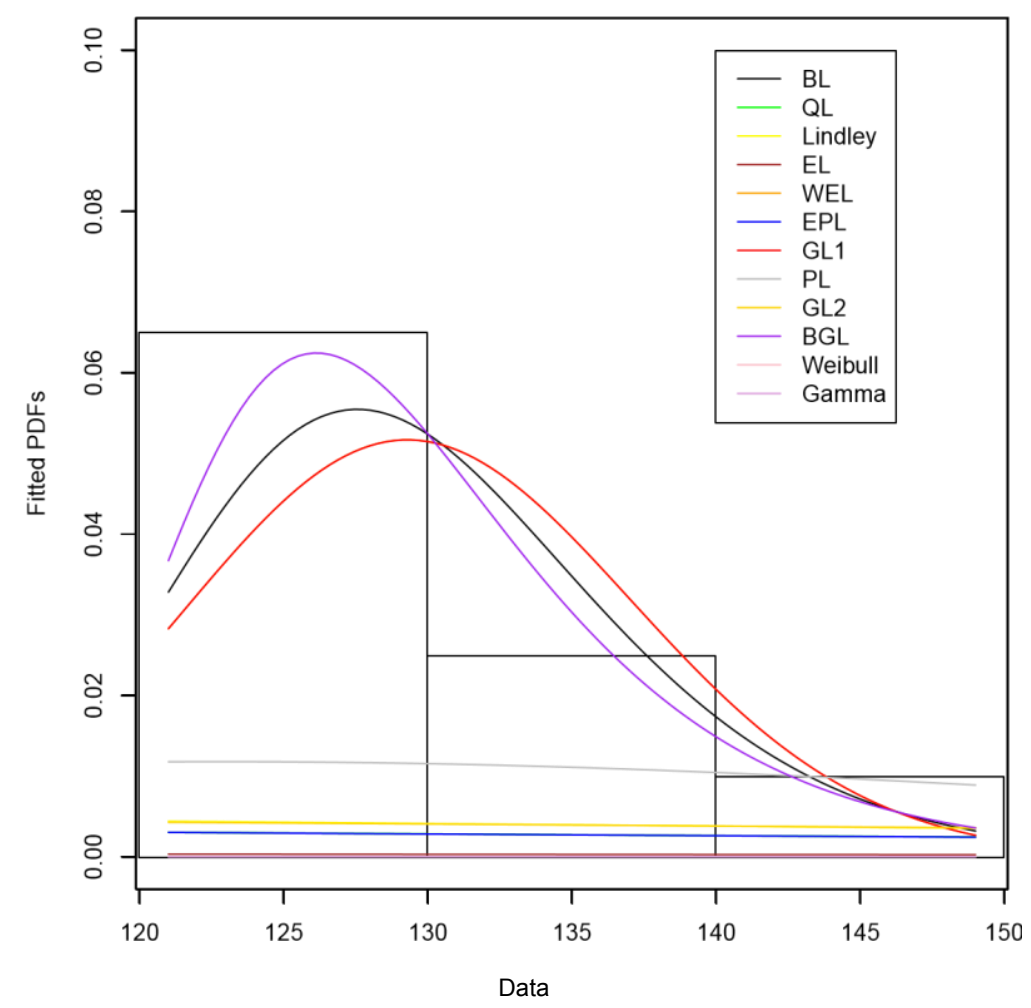


Figure 5: Histogram of the IQ data and the fitted pdfs of the BL, QL, Lindley, GL1, WEL, EL, EPL, PL, GL2, BGL, Weibull and gamma distributions.

\section{Appendix: $\mathbf{R}$ codes}

Here, we present $\mathrm{R}$ functions for computing the quantile function (Section 2.2), moments (Section 2.5), mean deviation about the mean (Section 2.7) and mean deviation about the median (Section 2.7). qf computes the quantile function, moments computes the $k$ th moment, mdmean computes the mean derivation about the mean and mdmedian computes the mean derivation about the median. The qf function makes use of the lambertWm1 function in the R contributed package lamW. This package must be installed before calling qf.

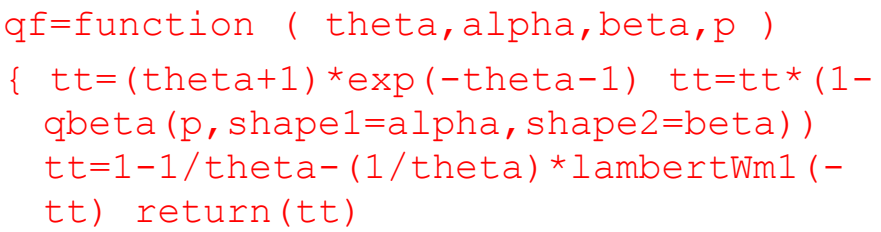




\begin{tabular}{|c|c|c|c|c|c|}
\hline Distribution & Estimates (ses) & -Log-likelihood & AIC & $\mathrm{BIC}$ & $p$-value \\
\hline Lindley & $\bar{\theta}=1.530 \times 10^{-2}\left(1.722 \times 10^{-3}\right)$ & 219.55 & 441.09 & 442.78 & 0.03 \\
\hline BL & $\begin{array}{l}\widehat{\alpha}=18330.5(18068.8), \\
\widehat{\beta}=3.189(1.422), \\
\widehat{\theta}=8.678 \times 10^{-2}\left(1.180 \times 10^{-2}\right)\end{array}$ & 136.36 & 278.72 & 283.78 & 0.28 \\
\hline QL & $\begin{array}{l}\widehat{\alpha}=9.999(3.692) \\
\widehat{\theta}=8.470 \times 10^{-3}\left(8.341 \times 10^{-3}\right)\end{array}$ & 219.69 & 443.38 & 446.76 & 0.02 \\
\hline EL & $\begin{array}{l}\widehat{\lambda}=9.522 \times 10^{-1}\left(4.856 \times 10^{-1}\right), \\
\widehat{\alpha}=-2.168 \times 10^{-6}\left(3.945 \times 10^{-6}\right), \\
\widehat{\beta}=1.671 \times 10^{-1}\left(6.111 \times 10^{-1}\right)\end{array}$ & 323.37 & 652.74 & 657.81 & 0.01 \\
\hline WEL & $\begin{aligned} \widehat{\theta} & =2.173\left(5.159 \times 10^{-1}\right), \\
\widehat{c} & =280.9(66.9)\end{aligned}$ & 138.50 & 281.00 & 284.38 & 0.11 \\
\hline EPL & $\begin{array}{l}\widehat{\theta}=12334.2(5924.1) \\
\widehat{\beta}=7.706 \times 10^{-3}\left(8.953 \times 10^{-2}\right)\end{array}$ & 234.63 & 473.25 & 476.63 & 0.01 \\
\hline GL1 & $\begin{array}{l}\widehat{\theta}=2.172(3.985), \\
\widehat{\alpha}=280.9(87.0), \\
\widehat{\gamma}=44.3(9.1)\end{array}$ & 138.50 & 283.00 & 288.07 & 0.06 \\
\hline PL & $\begin{array}{l}\widehat{\alpha}=2.885(3.411), \\
\widehat{\beta}=1.545 \times 10^{-6}\left(5.697 \times 10^{-4}\right)\end{array}$ & 177.96 & 359.93 & 363.31 & 0.03 \\
\hline GL2 & $\begin{array}{l}\widehat{\lambda}=9.507 \times 10^{-2}\left(4.231 \times 10^{-3}\right) \\
\widehat{\alpha}=13780.7(6476.5)\end{array}$ & 145.15 & 294.30 & 297.68 & 0.04 \\
\hline BGL & $\begin{array}{l}\widehat{a}=24555.3(28911.0), \\
\widehat{b}=9.299 \times 10^{-1}\left(2.423 \times 10^{-1}\right), \\
\widehat{\theta}=1.846 \times 10^{-1}\left(1.578 \times 10^{-2}\right), \\
\widehat{\alpha}=23772.5(28049.0)\end{array}$ & 136.28 & 280.56 & 287.31 & 0.25 \\
\hline Weibull & $\begin{array}{l}\widehat{a}=15.8(1.955), \\
\widehat{b}=7.482 \times 10^{-3}\left(7.872 \times 10^{-5}\right)\end{array}$ & 144.00 & 292.00 & 295.38 & 0.06 \\
\hline Gamma & $\begin{array}{l}\widehat{a}=281.9(83.0), \\
\widehat{b}=4.602 \times 10^{-1}\left(1.356 \times 10^{-1}\right)\end{array}$ & 138.50 & 281.00 & 284.38 & 0.11 \\
\hline
\end{tabular}

Table 1: Parameter estimates, standard errors, log-likelihood values and goodness of fit measures. 

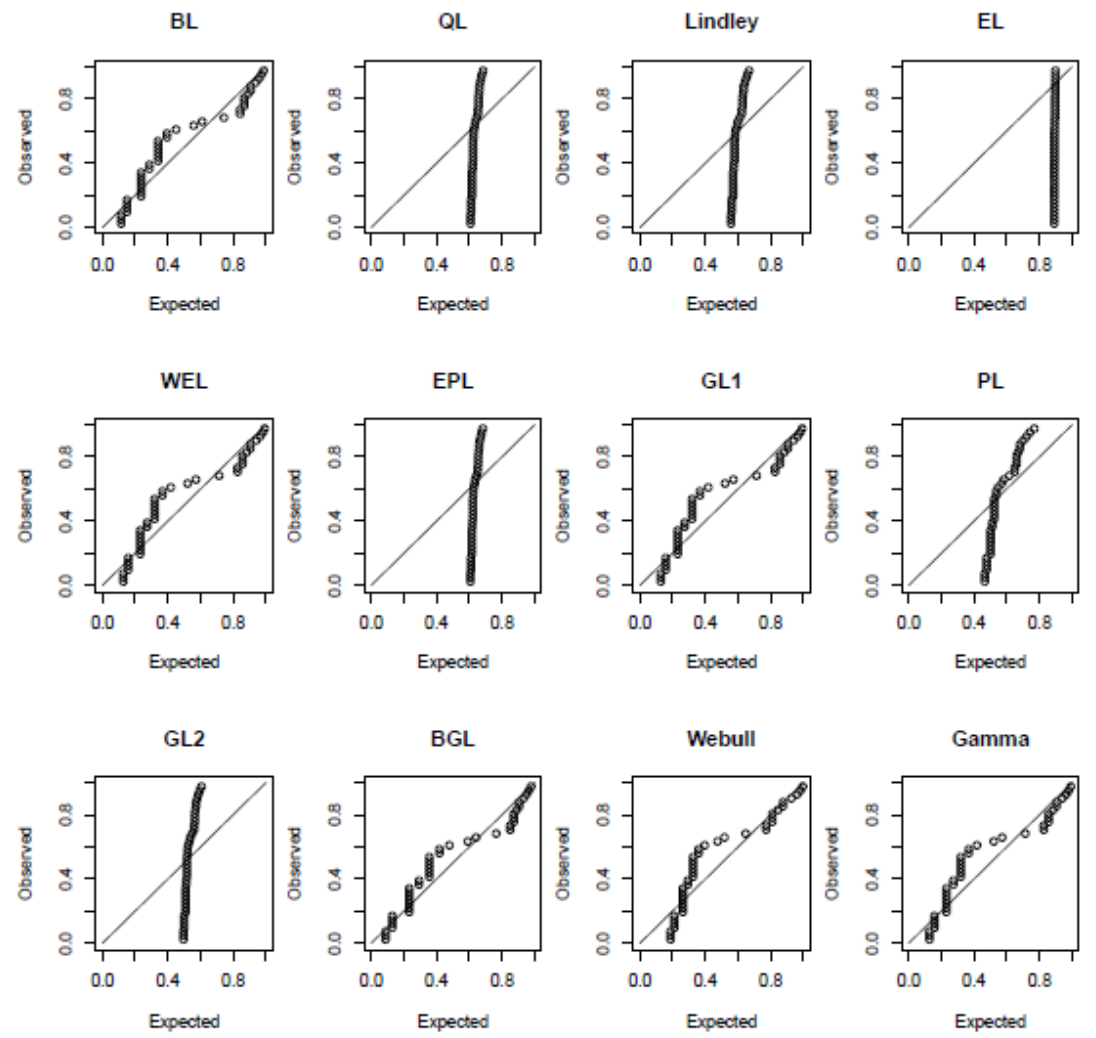

Figure 6: Probability plots for the fits of the BL, QL, Lindley, GL1, WEL, EL, EPL, PL, GL2, BGL, Weibull and gamma distributions.

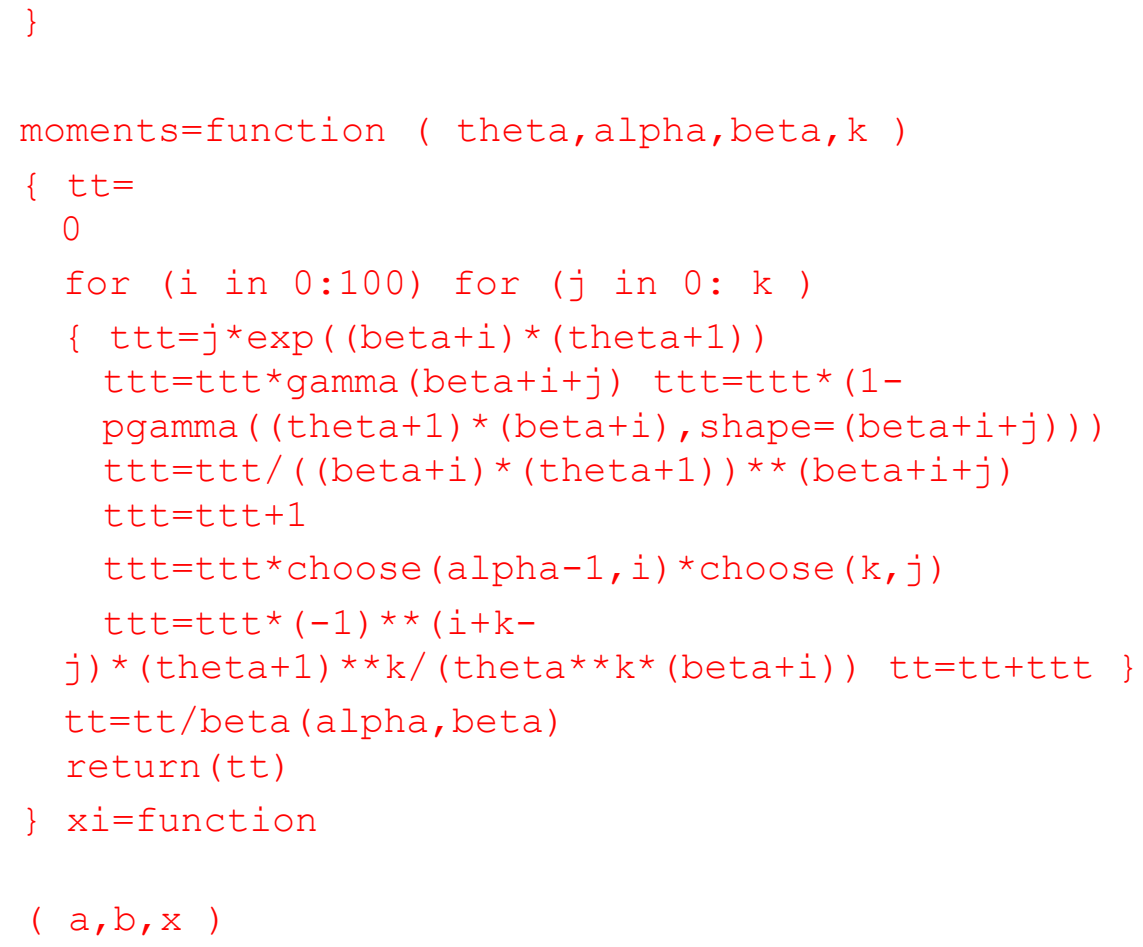




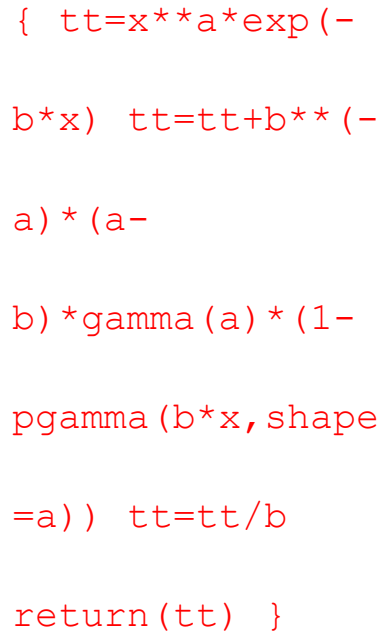




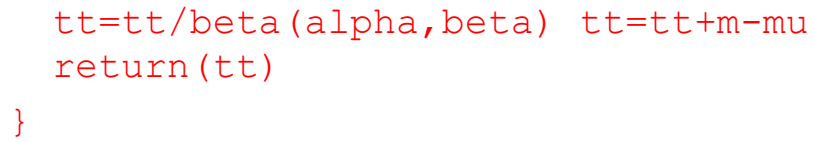

\section{Acknowledgments}

The authors would like to thank the Editor and the referee for careful reading and comments which greatly improved the paper.

\section{References}

[1] A. Akinsete, F. Famoye, C. Lee, The beta-Pareto distribution, Statistics 42 (2008) 547-563.

[2] H. S. Bakouch, B. M. Al-Zahrani, A. A. Al-Shomrani, V. A. A. Marchi, F. Louzada, An extended Lindley distribution, Journal of the Korean Statistical Society 41 (2012) 75-85.

[3] W. Barreto-Souza, H. S. Bakouch, A new lifetime model with decreasing failure rate, Statistics 47 (2013) 465-476.

[4] F. Chapeau-Blondeau, A. Monir, Evaluation of the Lambert $W$ function and application to generation of generalized Gaussian noise with exponent 1/2, IEEE Transactions on Signal Processing 50 (2002) 2160-2165.

[5] N. Eugene, C. Lee, F. Famoye, Beta-normal distribution and its applications, Communications in Statistics-Theory and Methods 31 (2002) 497-512.

[6] F. Famoye, C. Lee, O. Olumolade, The beta Weibull distribution, Journal of Statistical Theory and Applications 4 (2005) 121-136.

[7] M. E. Ghitany, D. K. Al-Mutairi, N. Balakrishnan, L. J. Al-Enezi, Power Lindley distribution and associated inference, Computational Statistics and Data Analysis 64 (2013) 20-33.

[8] M. E. Ghitany, F. Alqallaf, D. K. Al-Mutairi, H. A. Husain, A two-parameter weighted Lindley distribution and its applications to survival data, Mathematics and Computers in Simulation 81 (2011) 1190-1201.

[9] E. Gomez-Deniz, M. A. Sordo, E. Calderin-Ojeda, The log-Lindley distribution as an alternative to the beta regression model with applications in insurance, Insurance: Mathematics and Economics 54 (2014) 49-57.

[10] I. S. Gradshteyn, I. M. Ryzhik, Table of Integrals, Series, and Products, sixth edition, San Diego, Academic Press, 2000.

[11] P. Jodra, Computer generation of random variables with Lindley or Poisson-Lindley distribution via the Lambert $W$ function, Mathematics and Computers in Simulation 81 (2010) 851-859.

[12] L. Kong, C. Lee, J. H. Sepanski, On the properties of beta-gamma distribution, Journal of Modern Applied Statistical Methods 6 (2007) 187-211. 
[13] L. E. Lehmann, G. Casella, Theory of Point Estimation, second edition, Springer, New York, 1998.

[14] D. V. Lindley, Fiducial distributions and Bayes' theorem, Journal of the Royal Statistical Society B 20 (1958) 102-107.

[15] E. Mahmoudi, The beta generalized Pareto distribution with application to lifetime data, Mathematics and Computers in Simulation 81 (2011) 2414-2430.

[16] S. Nadarajah, H. S. Bakouch, R. Tahmasbi, A generalized Lindley distribution, Sankhy ${ }^{-}$a B 73 (2011) 331-359.

[17] S. Nadarajah, S. Kotz, The beta exponential distribution, Reliability Engineering and System Safety 91 (2006) 689-697.

[18] B. O. Oluyede, T. Yang, A new class of generalized Lindley distributions with applications, Journal of Statistical Computation and Simulation 85 (2015) 2072-2100.

[19] R Development Core Team, R: A Language and Environment for Statistical Computing, R Foundation for Statistical Computing, Vienna, Austria, 2014.

[20] T. G. Ramires, E. M. M. Ortega, G. M. Cordeiro, G. G. Hamedani, The beta generalized halfnormal geometric distribution, Studia Scientiarum Mathematicarum Hungarica 50 (2013) 523-554.

[21] R. Shanker, A. Mishra, A quasi Lindley distribution, African Journal of Mathematics and Computer Science Research 6 (2013) 64-71.

[22] M. D. Ugarte, A. F. Militino, A. T. Arnholt, Probability and Statistics with R, Chapman and Hall/CRC, London, 2008.

[23] H. Zakerzadeh, A. Dolati, Generalized Lindley distribution, Journal of Mathematical Extension 3 (2009) 13-25.

Received April 15, 2015; accepted Jane 19, 2015

S.M.T.K. MirMostafaee, M. Mahdizadeh ,Saralees Nadarajah

Department of Statistics, Faculty of Mathematical Sciences, University of Mazandaran, P.O. Box 47416-1467,

Babolsar, Iran

2Department of Statistics, Hakim Sabzevari University, P.O. Box 397, Sabzevar, Iran

3School of Mathematics, University of Manchester, Manchester M13 9PL, UK 
